\title{
The Impact of Helicopter Emergency Medical Service Night Operations in South East England
}

\author{
Leigh Curtis, MSc ${ }^{1}$, Mark Salmon, MBChB ${ }^{1}$, Richard M. Lyon, MBChB, MD ${ }^{1,2, *}$ \\ ${ }^{1}$ Kent, Surrey and Sussex Air Ambulance Trust, Kent, UK \\ ${ }^{2}$ University of Surrey, Surrey, UK
}

\begin{abstract}
A B S T R A C T
Objective: This study sought to assess the impact of a helicopter emergency medical service (HEMS) capable of night operations.

Methods: This is a retrospective case review of all night HEMS missions attended by a charity air ambulance service in South East England over a 2-year period (October 1, 2013, to October 1, 2015).

Results: During the 2-year trial period, the HEMS service undertook a total of 5,004 missions and attended to 3,728 patients. Of these, 1,373 missions, or 27.4\% of the total HEMS activity, were night missions. Night missions increased from year $1(n=617)$ to year $2(n=756)$. A mean of 1.9 missions per night were conducted, resulting in the treatment of 1.3 patients per night. A higher proportion of patients were transported to a major trauma center at night $\left(64 \%\right.$ vs. $\left.51 \%, \chi^{2}=41.8, P<.0001\right)$. Weather conditions prevented HEMS from responding at night via air for $15 \%$ of the night operational hours.

Conclusion: A 2-year trial period of a night HEMS service in South East England showed the predicted activation rate, with a mean of 1.3 patients attended to per night. Patients transported to a major trauma center had a mean Injury Severity Score of 23. Further research is warranted to determine if the night HEMS service conveys a patient outcome benefit.
\end{abstract}

Copyright @ 2017 Air Medical Journal Associates. Published by Elsevier Inc. All rights reserved.

Major trauma is a leading cause of serious morbidity and mortality. ${ }^{1,2}$ Advanced prehospital care can improve the outcome for major trauma patients. ${ }^{3,4}$ Kent, Surrey \& Sussex Air Ambulance Trust (KSSAAT) delivers advanced prehospital care by deploying 2 doctor/paramedic teams by aircraft or response car. The 2 teams respond from 2 separate bases in South East England, 1 based in Surrey and 1 based in Kent. Historically, KSSAAT was operational between 0700 and 1900 hours 7 days per week. However, major trauma frequently occurs overnight, and the lack of a night helicopter emergency medical service (HEMS) was felt to be detrimental to enhanced patient care because no enhanced prehospital medical care was available overnight.

Before any night HEMS service was available, KSSAAT undertook a prospective study in 2010 to explore the possible impact that a night HEMS service may have. ${ }^{5}$ This study showed the likely need of a night HEMS team being tasked 1.7 times per night during the hours of 1900 to 0700 . The incidence of these predicted cases continued throughout the entire night period but with gradually decreasing frequency. Most

\footnotetext{
* Address correspondence to: Richard M. Lyon, MBChB, MD, Kent, Surrey E Sussex Air Ambulance Trust, Wheelbarrow Park Estate, Pattenden Lane, Marden, Kent TN12 9QJ, UK. E-mail address: richardl@kssairambulance.org.uk (R.M. Lyon).
}

nights of the week were predicted to have similar levels of activity with the exception of Saturday, which appeared to be the busiest night of the week. A high number of the cases identified resulted in the patient being transported to a major trauma center (MTC), indicating that HEMS activation may well have been warranted.

Based on this study, KSSAAT made the decision to commit itself to exploring the options for night HEMS operations. This commitment was the start of a 3-year research, development, and training process, which culminated with the launch of a 2-year night HEMS operational trial on the night of September 26, 2013. At this point, KSSAAT became the first 24/7 helicopter-based HEMS in the United Kingdom. The purpose of this study is to review the activity, case mix, and demographics of the 2-year night HEMS trial period. We sought to compare the actual activity of the night HEMS service with the previously estimated need.

\section{Methods}

The region that KSSAAT serves has a resident population of over 4.5 million people spread over an area of approximately $9,000 \mathrm{~km}^{2}$ (3,600 square miles). Based on prolonged ( $>1.5$ hours in some cases) time to reach incidents within this area, it was felt that an HEMS service was warranted over a land-based response model. 
To comply with Clean Air Act regulations ${ }^{6}$ and to deploy the safest possible night HEMS service, a new aircraft (MD902, McDonnellDouglas helicopters) was deployed. This aircraft was operated by 2 pilots and fully equipped for night flight with the necessary instrument flight certification, traffic collision avoidance system, search light, wire cutters, and bespoke navigation equipment. In addition, all members of the crew (including doctors and paramedics) wore night vision goggles. During the mission planning phase of every activation, an ad hoc site was identified as close to the incident location as possible. In addition, presurveyed landing sites across the region were used, such that every mission always had a secondary, presurveyed site as a backup.

All cases of night HEMS activation are recorded on a bespoke electronic database (HEMSbase; MedicOne Systems Ltd, Surrey, UK). All activations during the hours of 1900 to 0700 were prospectively recorded. We analyzed all HEMS missions from October 1, 2013, to October 1, 2015. This study met National Institute for Healthcare Research guidelines as a service evaluation, and, therefore, formal ethical approval was not required.

\section{Results}

Missions and Patient Contact

During the 2-year trial period of this study, KSSAAT performed a total of 5,004 missions and attended to 3,728 patients. Between year 1 and year 2, we experienced a 7.5\% growth in activity (year 1: 2,412 vs. year 2: 2,592 missions) and a $4.2 \%$ rise in patient numbers. Of this activity, 1,373 missions (27.4\% of the total HEMS activity) were night missions. These missions resulted in KSSAAT treating 942 patients, which is equal to $25.3 \%$ of our patient total. Night missions increased from year $1(n=617)$ to year $2(\mathrm{n}=756)$.

We predicted that KSSAAT activity levels at night would be approximately 1.7 missions per night. ${ }^{5}$ The experience of the 2-year trial is that activity levels equated to an average of 1.9 missions per night, resulting in the treatment of 1.3 patients per night. Activity at night grew across the 2-year period, resulting in year 1 performing an average of 1.7 missions and treating 1.2 patients per night and in year 2 an average of 2.1 missions and 1.4 patients per night. Although there were month-by-month peaks and troughs in activity, the overall trend was indicative of a continuing growth in night activity, as shown in Figure 1.

\section{Day of the Week}

The busiest nights of the week were found to be Saturday and Sunday, as shown in Figure 2.

\section{Time of Night}

Two thirds of the activity occurred in the first half of the night shift, before 0100 hours, with the remaining third falling in the second half of the shift. Activity decreased across the night evenly such that in the first 4 hours of the night shift we performed on average 1 mission per night; in the second 4 hours, we performed a little over 1 mission every other night; and in the final 4 hours, we performed 1 mission every third night (Fig. 3).

\section{Patient Population}

A higher proportion of younger (15-30 years) patients were encountered by night. The average age of night patients was 37.8 years old, 4.4 years younger than the average patient we treat by day.

\section{Mission Type}

The median night planning phase from activation to launch was noted to be 14 minutes. We observed a difference in the type of incidents attended by night compared with those attended by day. Although road traffic collision was by day the leading cause of activation, it was at a significantly reduced rate. Most notable among night patients were the significantly increased rates of victims of assault and self-harm compared with the day patient cohort.

\section{Patient Acuity}

A surrogate marker for injury severity in the prehospital phase is whether patients are conveyed directly to an MTC. After the introduction of the night HEMS service, a significantly higher proportion (64\% vs. $51 \%, \chi^{2}=41.8, P<.0001$ ) of patients were conveyed directly to an MTC compared with during daytime operations. The mean Injury Severity Score for patients transported to an MTC by night was higher than those transported during the day ( 23.5 vs. $22.0, P<.05$ ).

A significant number of severely injured (> Injury Severity Score 15) patients received enhanced prehospital care, with a higher proportion of severely injured patients being encountered at night than during the day. Having an HEMS service available by night allowed enhanced medical care to be delivered to the scene more rapidly than by land response.

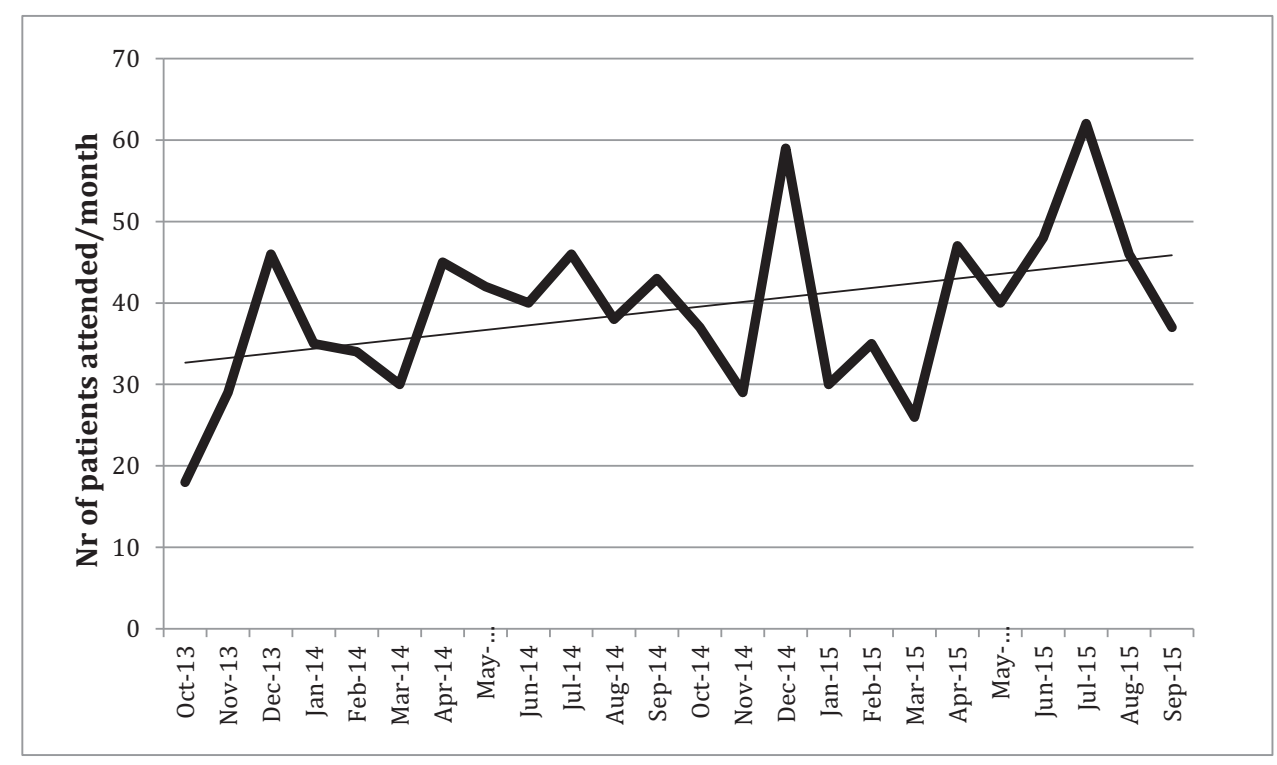

Figure 1. The night activity during the trial period by 24 linear months. 


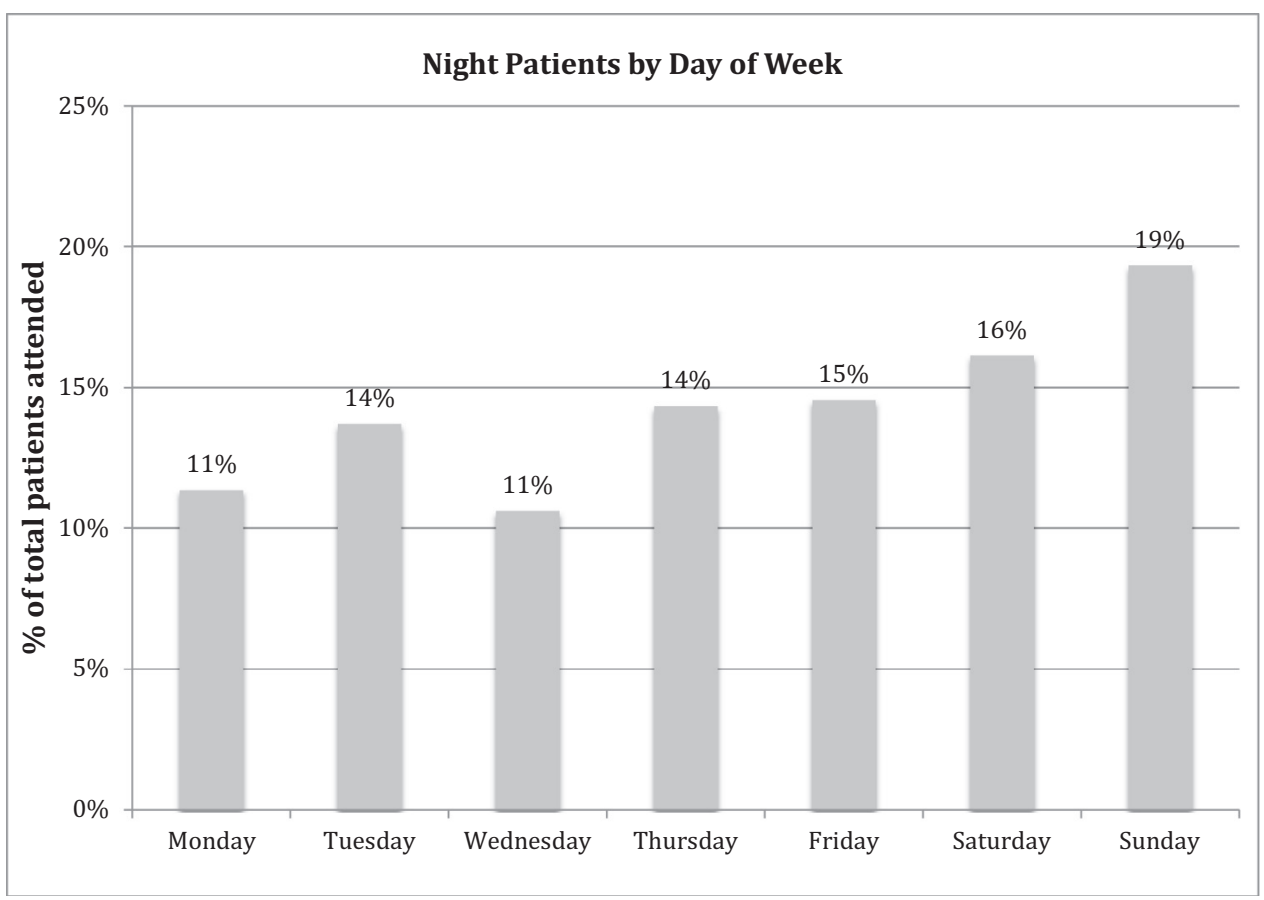

Figure 2. The activity during the trial period by the day of the week.

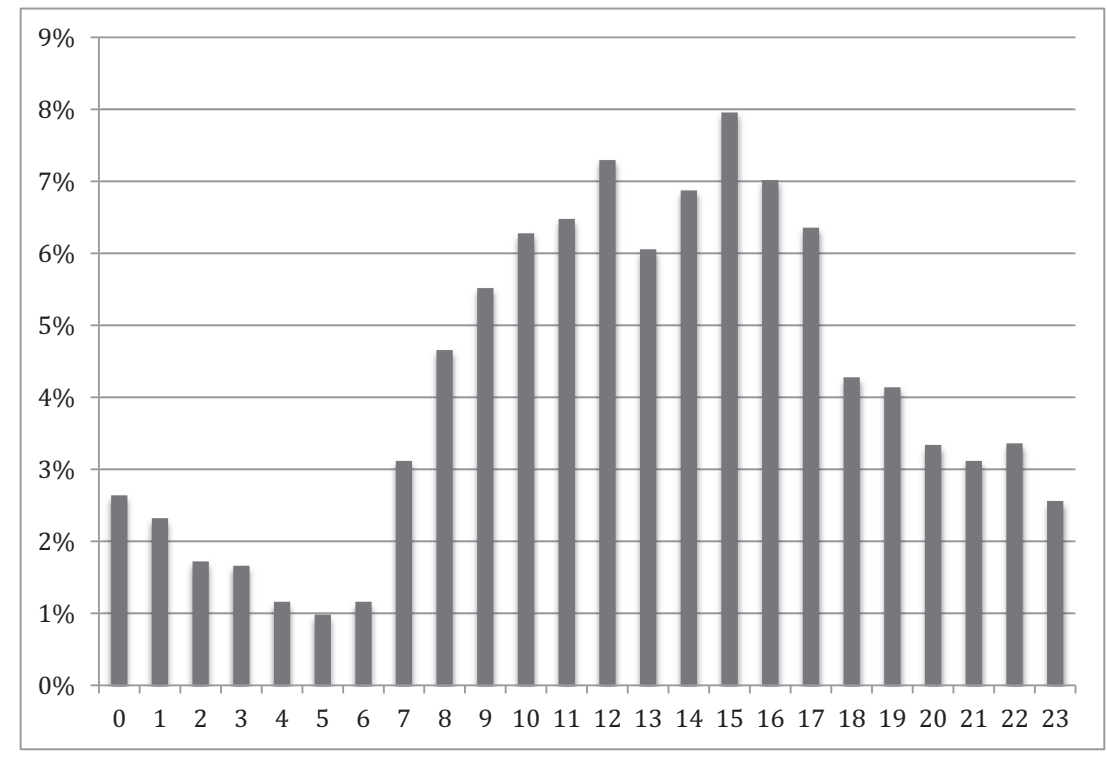

Figure 3. The percentage of activity by the hour of day.

\section{Weather}

Weather conditions prevented KSSAAT from responding at night via air for $15 \%$ of the night operational hours (Fig. 4). The winter months resulted in the highest proportion of time when the weather was below the minimum requirements for night HEMS operations.

\section{Discussion}

This operational trial of providing an HEMS service by night showed that the previously expected demand ${ }^{5}$ was found to be real. Activity grew across the study period, and if current growth rates continue, night operations would represent one third of all KSSAAT missions. The night demand is relatively predictable and continues throughout the night and year-round. The patients encountered by night are relatively young, often have subtly different mechanisms of injury with differing treatment priorities, and are commonly severely injured. Other regions in the United Kingdom and further afield are likely to have similar populations and incidence of trauma. This study highlights that a predicted need for a night HEMS service was met, and other regions may wish to explore their local need and consider expanding services that currently only operate by day.

Previous studies ${ }^{7,8}$ have shown a similar demographic of a higher proportion of road traffic collisions occurring by night and also 


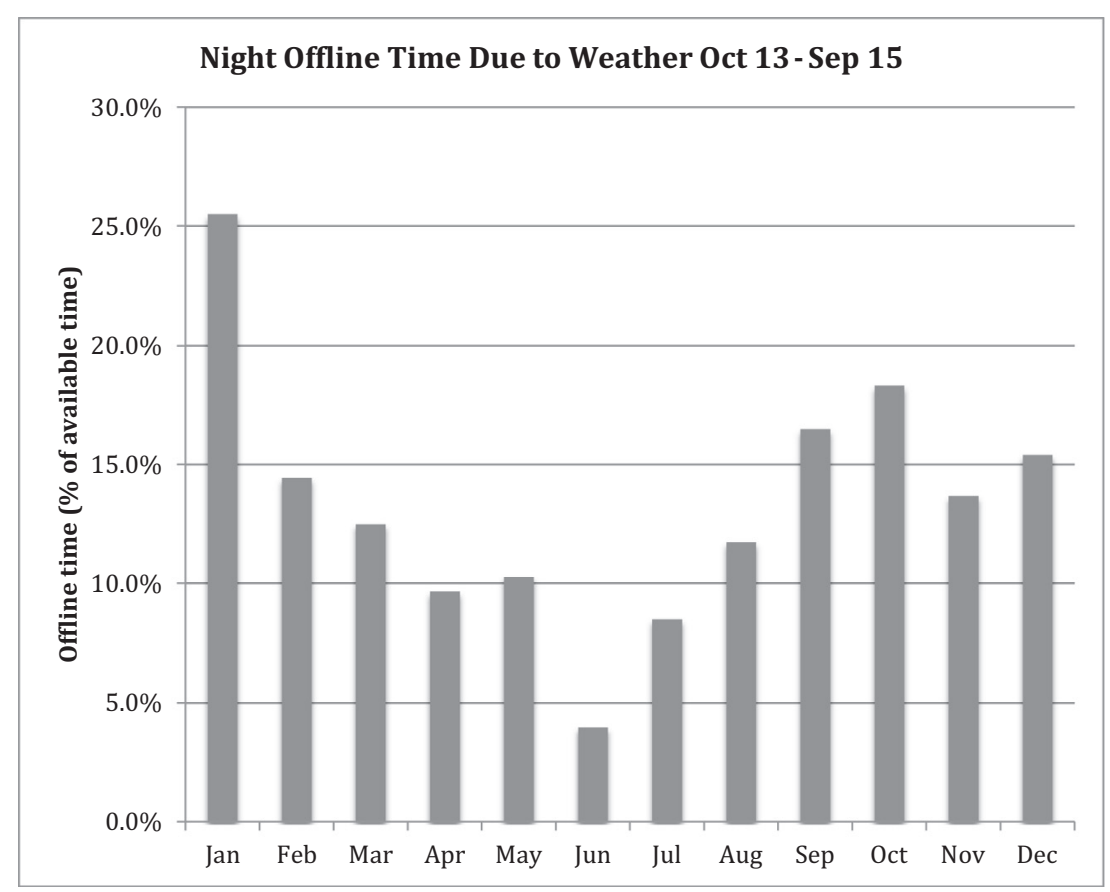

Figure 4. Night off-line time because of weather restrictions.

assaults. These assault victims may suffer blunt or penetrating trauma that may require enhanced medical care. Given the need to plan a night mission, it is important that potential missions are identified as early as possible to enable the crew to launch in a timely but safe fashion. We found that early activation and launch with a stand-down during the flight phase if HEMS was not required ensured timely activation.

Weather restrictions can play a significant part in night HEMS operations. In the United Kingdom, the minimum requirements for a night HEMS mission are a cloud base of $1,200 \mathrm{ft}$ above ground level and horizontal visibility of $3,000 \mathrm{~m}$. Over the winter months in the United Kingdom, weather minima were frequently encountered, resulting in the enhanced care team having to respond via car. This led to significant delays in reaching patients compared with using the aircraft. Other countries use, or are developing, advanced aircraft navigation systems such as Global Positioning System point in space technology to safely operate HEMS in degraded meteorologic conditions. The exploration of point in space in the United Kingdom may potentially significantly increase the proportion of time, particularly during winter months, when night HEMS could operate.

This study has several limitations. We have only been able to quantify the demographic of the patients attended by the HEMS service during the trial. The study was unable to examine all major trauma patients from the region that occurred during the same time period, in particular those attended by the regional ambulance service but not by HEMS. Seasonal variation does occur in HEMS services, and it would be important to continue to collate these data over a longer time period, particularly looking for activity trends. Further data would be useful to compare the acuity of patients transported at night versus day and by HEMS versus non-HEMS services. This study did not attempt to explore the impact of patient outcome for those patients attended by the HEMS service. Further research is warranted to examine the time savings from the incident occurring to the delivery of enhanced prehospital care and time to arrival at an MTC. In addition, research is warranted to determine if the night HEMS service conveys patient outcome benefit.

\section{Conclusion}

A 2-year trial period of a night HEMS service in South East England showed the predicted activation rate of 1.3 patients attended to per night, with those transported to an MTC being more severely injured than those transported by day. More assaults were encountered by night. The use of a night vision-equipped, Instrument Flight Rules-capable MD902 aircraft allowed the night HEMS trial to be completed successfully. Poor weather prevented a significant number of missions from being conducted by air, particularly during the winter. Further research is warranted to determine if the night HEMS service conveys a patient outcome benefit.

\section{References}

1. Søreide K, Krüger AJ, Vårdal AL, et al. Epidemiology and contemporary patterns of trauma deaths: changing place, similar pace, older face. World J Surg. 2007;31:2092-2103.

2. Cothren CC, Moore EE, Hedegaard HB, et al. Epidemiology of urban trauma deaths: a comprehensive reassessment 10 years later. World J Surg. 2007;31:1507-1511.

3. Brown JB, Gestring ML, Guyette FX, et al. Helicopter transport improves survival following injury in the absence of a time-saving advantage. Surgery. 2016;159:947-959.

4. Funder KS, Rasmussen LS, Lohse N, et al. Long-term follow-up of trauma patients before and after implementation of a physician-staffed helicopter: a prospective observational study. Injury. 2016;47:7-13.

5. Lyon RM, Vernon J, Nelson M, et al. The need for a UK helicopter emergency medical service by night: a prospective, simulation study. Air Med J. 2015;34:195198.

6. Civil Aviation. Safety Directive 201. Available at: https://www.caa.co.uk/docs/33/ SafetyDirective2012004.pdf. Accessed May 1, 2014.

7. Hesselfeldt R, Steinmetz J, Jans H, et al. Impact of a physician-staffed helicopter on a regional trauma system: a prospective, controlled, observational study. Acta Anaesthesiol Scand. 2013;57:660-668.

8. Østerås $\varnothing$, Brattebø G, Heltne JK. Helicopter-based emergency medical services for a sparsely populated region: a study of 42,500 dispatches. Acta Anaesthesiol Scand. 2016;60:659-667. 